\title{
GTI-2040 displays cooperative anti-tumor activity when combined with interferon $\alpha$ against human renal carcinoma xenografts
}

\author{
AIKATERINI VASSILAKOS, YOON LEE, STÉPHANE VIAU, \\ NINGPING FENG, HONGNAN JIN, VIENGTHONG CHAI, \\ MING WANG, TINA AVOLIO, JIM WRIGHT and AIPING YOUNG
}

Lorus Therapeutics Inc., 2 Meridian Rd., Toronto, Ontario M9W 4Z7, Canada

Received July 18, 2008; Accepted September 22, 2008

DOI: 10.3892/ijo_00000126

\begin{abstract}
GTI-2040, an antisense oligonucleotide targeting the small subunit of ribonucleotide reductase, acts as an anti-tumor agent in animal models of human cancer. In the present study, the anti-tumor activity of GTI-2040, in combination with interferon $\alpha(\operatorname{IFN} \alpha)$ was investigated against human renal cell carcinoma tumors xenografted into mice. The human renal cell carcinoma cell lines, Caki-1 and A498 were sensitive to IFN $\alpha$ both in vitro and when implanted into mice. In combination with GTI-2040 there were cooperative effects at intermediate doses of the two agents and complete tumor regression at higher combination doses. A control oligonucleotide was not effective as a monotherapy and did not improve the efficacy of IFN $\alpha$. The effect of combination treatment on apoptosis and proliferation of tumor cells, isolated from xenografted tumors, was examined by histochemistry. GTI-2040 increased the percentage of cells undergoing apoptosis with a concomitant decrease in proliferation. IFN $\alpha$ alone had no effect but in combination with GTI-2040 resulted in increased apoptosis and decreased proliferation compared to GTI-2040 alone. Taken together these results expand the potential clinical applications of GTI-2040 to include combination therapy with IFNa.
\end{abstract}

Correspondence to: Dr Yoon Lee, Lorus Therapeutics Inc., 2 Meridian Road, Toronto, Ontario M9W 4Z7, Canada

E-mail: ylee@lorusthera.com

Abbreviations: RNR, ribonucleotide reductase; R2, ribonucleotide reductase subunit 2 ; R1, ribonucleotide reductase subunit 1 ; ODN, oligodeoxynucleotide; AS-ODN, antisense oligodeoxynucleotide; PS-ODN, phosphorothioate oligodeoxynucleotide; RCC, renal cell carcinoma; IFN $\alpha$, interferon $\alpha$

Key words: ribonucleotide reductase, antisense oligonucleotides, immunotherapy

\section{Introduction}

It is estimated that in 2008 there will be 54,390 new cases of renal cancer in the US with $\sim 13,010$ deaths (1). Renal cell carcinoma (RCC) is the most commonly diagnosed renal malignancy accounting for $85-90 \%$ of cases $(1,2)$. If diagnosed at the very early stages most forms of RCC can be treated by surgery and in some cases radiation. In contrast, the prognosis for advanced disease is poor with 5-year survival rates currently assessed at $<10 \%(1,2)$. Once RCC has progressed there are few effective treatment options. RCC has been largely resistant to chemotherapy with chemotherapeutic agents such as vinblastine and FU demonstrating $<10 \%$ response rates in clinical trials $(3,4)$. High dose IL-2 results in response rates in the range of $10-20 \%$ with lasting response of $\sim 5-10 \%(5,6)$. As a result, IL-2 has become the standard of care for renal cell carcinoma. Although a significant advancement in treatment, high-dose IL-2 is limited by toxicity resulting in only a small subset of patients being candidates for treatment $(2,5,7)$. Due to the limited patient population, eligible for high-dose IL-2 therapy, interferon $\alpha$ (IFN $\alpha$ ) has also emerged as a standard treatment and as a preferred comparator arm in phase III clinical studies evaluating newer drugs $(7,8)$. In the past few years, promising new drug candidates have been developed based on emerging information on the genetic and molecular pathways involved in development of RCC $(9,10)$. These include anti-VEGF monoclonal antibodies (bevacizumab), multi-kinase inhibitors (sorafenib, sunitinib) and inhibitors of the mammalian target of rapamycin (mTOR) pathway (temsirolimus) (11-13). A number of these drug candidates have demonstrated clinical activity with some studies indicating efficacy superior to immunotherapy (11-13). Although some progress has been made in the treatment of renal cell carcinoma there remains a considerable need to develop drugs with good safety profiles that can be added to existing treatments, both standard immunotherapy and emerging treatments.

Ribonucleotide reductase (RNR) catalyzes the reaction in which 2'-deoxyribonucleotides are synthesized from the corresponding ribonucleoside 5 '-diphosphates $(14,15)$. This step is the rate-limiting reaction in the production of 2'deoxyribonucleoside 5'-triphosphates required for DNA 
replication and repair. RNR consists of two protein components. $\mathrm{R} 1$ is a $160-\mathrm{kDa}$ dimer that contains at least two different effector-binding sites and $\mathrm{R} 2$ is a $78-\mathrm{kDa}$ dimer that contains a nonheme iron that participates in catalysis by forming an unusual free radical on the aromatic ring of a tyrosine residue $(16,17)$. Expression of both $\mathrm{R} 1$ and $\mathrm{R} 2$ are required for enzymatic activity. $\mathrm{R} 1$ in proliferating cells is relatively constant throughout the cell cycle, while R2 expression varies with elevated R2 levels observed during late G1 or early S-phase, suggesting that expression of R2 plays a role in regulation of RNR activity, DNA synthesis and cell proliferation (18-24). Recently, an R2 homologue, p53R2, was identified. The human p53R2 gene contains a p53 binding site and is $\sim 80 \%$ identical to R2 $(25,26)$. The role of p53R2 is unique from $\mathrm{R} 2$ in that it is induced upon exposure to UV light, $\gamma$-irradiation or DNA-damaging agents and supplies the dNTPs necessary for DNA repair $(26,27)$. Like R2, p53R2 appears to complex with R1 to form an active RNR (28). RNR activity has been correlated with cancer progression in a number of studies $(29,30)$ and several therapeutic agents are currently used which inhibit RNR as part of their mechanism of action. These include Hydroxyurea (Hydrea ${ }^{\circledR}$, Bristol-Myers Squibb and Hydroxyurea Capsules, Roxane), Gemcitabine $\left(\right.$ Gemzar $^{\circledR}$, Eli Lilly) and Fludarabine (Fludara ${ }^{\circledR}$, Berlex). These drugs are limited in large part by the development of drug resistance.

In addition to its role in RNR activity, the $\mathrm{R} 2$ protein, independent of $\mathrm{R} 1$, appears to play a role in determining the malignant potential of cells via synergism with a number of activated oncogenes $(31,32)$. Altered R2 gene expression cooperates with ras in mechanisms of malignant progression $(31,32)$. Increased expression of R2 has been found to increase the drug-resistant properties of cancer cells while a retrovirus vector containing the $\mathrm{R} 2$ sequence in an antisense orientation in these cells has been demonstrated to reverse such drug resistance in vitro (33-37). Overexpression of R2 in KB carcinoma cells increases invasive potential as measured by in vitro migration of cultured cells (36). In addition, transfection of an IPTG inducible antisense cDNA against R2, but not $\mathrm{R} 1$, resulted in decreased proliferation of $\mathrm{KB}$ carcinoma cells in vitro (38). These studies would indicate that, apart from the anti-proliferative effect of RNR inhibition, the specific inhibition of the $\mathrm{R} 2$ protein would likely provide additional therapeutic benefit.

Several pre-clinical studies support targeting of R2 in combination with cytotoxic chemotherapeutics. Drugs that inhibit RNR, as part of their mechanism of action (hydroxyurea, FU, trimidox, triapine) have been shown to increase sensitivity to other drugs (39-43). In addition, recent studies have demonstrated that decreasing RNR R2 expression via siRNA treatment results in increased sensitivity to gemcitabine both in vitro and in vivo $(44,45)$. Taken together these results suggest that there may be several mechanisms by which targeting of R2 may increase sensitivity to anti-cancer drugs.

Antisense oligonucleotides (AS-ODN) are short DNA molecules that can interfere with gene expression by forming duplexes with complementary sequences of target messenger RNAs (mRNAs) (46). A previous study screened 102 oligonucleotides (ODNs) complementary to RNR subunit
R2 for the ability to decrease R2 mRNA levels in vitro (47). One ODN, GTI-2040, was further characterized and demonstrated specific, dose-dependent down-regulation of R2 with a concomitant decrease in proliferation, tumor growth and metastasis. In particular, GTI-2040 demonstrated excellent activity against two human renal cell carcinoma tumors implanted in mice. A completed phase I trial of GTI-2040 for the treatment of advanced cancers demonstrated a favorable safety profile at therapeutically relevant doses (48) and GTI-2040 is currently being evaluated in clinical trials in a number of cancer indications. A recently completed phase II combination study of GTI-2040 with capecitabine for renal cell carcinoma demonstrated a reasonable toxicity profile and a high incidence of disease stabilization. However, subsequent to initiation of this study capecitabine has been found to lack significant activity for treatment of renal cell carcinoma (49). This suggests that GTI-2040 should be tested in combination with an agent that has clinically proven activity for treatment of renal cell carcinoma.

In the present study, the potential of GTI-2040 as an antitumor drug candidate for treatment of renal cell carcinoma, was examined further. The anti-tumor activity of GTI-2040 in combination with IFN $\alpha$ was tested against human RCC cell lines xenografted into mice. The activities were compared to treatment with GTI-2040, alone and in combination with IFNa. Finally, in an effort to understand the mechanism by which GTI-2040 acts in combination with IFNa, cell proliferation and apoptosis in xenograft tumor samples were studied by immunohistochemistry.

\section{Materials and methods}

Oligonucleotides. All oligonucleotides used in this study were fully thioated. They were synthesized on an automated DNA synthesizer (Perkin-Elmer, USA) by Boston BioSystem Inc. (Boston, MA). Oligonucleotides were purified and purity assessed by reversed-phase high performance liquid chromatography. Each oligonucleotide preparation was found to be $>95 \%$ full-length material. GTI-2040 hybridizes to coding region of R2 mRNA. A scrambled control analogue of GTI-2040, GTI-2040Scr, is not complementary to R2, while maintaining the same base composition ratio (47).

Cell lines and cell culture. Unless noted otherwise in the text human tumor cell lines were purchased from the American Type Culture Collections (ATCC) (Rockville, MD). Caki-1 and A498 cell lines were maintained, according to ATCC recommendation, in $\alpha-\mathrm{MEM}$ or McCoy's 5 a medium (Gibco BRL, Gaithersburg, MD) supplemented with 10-20\% fetal calf serum (FCS) at $37^{\circ} \mathrm{C}$ in a humidified atmosphere containing $5 \% \mathrm{CO}_{2}$. All media used in these experiments also contain $100 \mathrm{x}$ diluted antibiotic solution that consist of $100 \mathrm{U} / \mathrm{ml}$ penicillin and $100 \mu \mathrm{g} / \mathrm{ml}$ streptomycin (Gibco BRL).

In vitro treatment with IFNa and XTT proliferation assay. In order to determine whether A498 and Caki-1 cells are sensitive to direct inhibition of cell proliferation by IFN $\alpha$, cells were grown in culture as described above and treated with increasing concentrations of IFN $\alpha$. Cells $\left(1 \times 10^{3}\right)$ per well were 
seeded onto 96-well plates and incubated overnight. After overnight incubation, the media were removed and fresh media containing $0,100,200,400,1000,3000$ and 10000 units IFN $\alpha$ per $\mathrm{ml}$ were added to each well as indicated. Each treatment was performed in triplicate and at the times indicated in the figure legend. Cells were incubated with XTT reaction solution according to the manufacturer's instructions (Roche, Cell Proliferation kit II) and the absorbance at 490 $\mathrm{nM}$ was measured using an ELISA plate reader. The results are presented as a \% of untreated control cells.

In vivo tumor growth assay. SCID mice were purchased from Charles River Laboratories, Montreal, Canada and the experiments were typically initiated when the mice were 6-7 weeks old. Human tumor cells were grown in appropriate growth medium and $3 \times 10^{6}-1 \times 10^{7}$ cells suspended in $100 \mu \mathrm{l}$ of PBS were subcutaneously injected into the right flank of the animals with \#23 gauge needle. Unless otherwise noted each experimental group typically contained 10 mice. After the size of tumor reached a mean tumor volume of $75-100 \mathrm{~mm}^{3}$, treatment was initiated as described in the figure legends. IFN $\alpha$ was purchased from (Schering-Plough, Canada).

Anti-tumor activity was estimated by the measurement of tumor volume with a caliper at two-day intervals. Tumor volume was calculated by a formula $1 \mathrm{X} \mathrm{w} \mathrm{X} \mathrm{h} / 2$, where 1 indicates length, w indicates width and $\mathrm{h}$ indicates height. Within $24 \mathrm{~h}$ after the last treatment, the animals were sacrificed and tumor and body weights were measured.

Immunohistochemistry. All procedures preceding the isolation of tumor samples were performed at Lorus Therapeutics. Subsequent immunohistochemistry and image analyses were performed at the Calcium Research Laboratory, Department of Medicine, McGill University, Montreal, Canada. The xenograft tumors were removed and fixed in PLP fixative (2\% paraformaldehyde containing $0.075 \mathrm{M}$ lysine and $0.01 \mathrm{M}$ sodium periodate solution) overnight at $4^{\circ} \mathrm{C}$. After fixation the samples were dehydrated in graded alcohols and embedded in low melting point paraffin and $5 \mu \mathrm{m}$ sections were cut on a rotary microtome. Paraffin sections were stained for macrophage and NK1.1 using the avidin-biotin-peroxidase complex technique, as described previously (50). Briefly, mouse anti-macrophage monoclonal antibody diluted 1:100 (Abcam) or mouse anti-mouse NK1.1 antibody diluted 1:100 (Caltag Laboratories) was applied to sections overnight at room temperature. As a negative control, the preimmune serum was substituted for the primary antibody. After washing with high salt buffer (50 mM Tris- $\mathrm{HCl}, 2.5 \% \mathrm{NaCl}, 0.05 \%$ Tween-20, pH 7.6) for $10 \mathrm{~min}$ at room temperature followed by two 10-min washes with Tris-buffered saline, the sections were incubated with secondary antibody (biotinylated goat anti-rat or mouse $\mathrm{IgG}$ ), washed as before and processed using the Vectastain ABC-AP kit (Vector Laboratories, Inc.). Red pigmentation to demarcate regions of immunostaining was produced by a 10- to 15-min treatment with Fast Red TR/ Naphthol AS-MX phosphate (Sigma, containing $1 \mathrm{mM}$ levamisole as endogenous alkaline phosphatase inhibitor). The sections were then washed with distilled water, counterstained with methyl green and mounted with Kaiser's glycerol jelly.
BrdU labeling of mice. 5-bromo2'-deoxy-uridine (BrdU, $100 \mathrm{mg} / \mathrm{kg}$ body weight) was injected intraperitoneally into mice $2 \mathrm{~h}$ prior to end of study and tumor collection. BrdUpositive cells were detected by immunohistochemistry using mouse anti-BrdU antibody (Sigma).

Detection of apoptotic cells. De-waxed paraffin sections were stained with an in situ cell death detection kit (Boehringer Mannheim), as previously described (51). Briefly, following treatment with $3 \mu \mathrm{g} / \mathrm{ml}$ of proteinase $\mathrm{K}$ for $20 \mathrm{~min}$ at room temperature, the sections were incubated with a reaction mixture for terminal deoxynucleotidyltransferase-mediated nick end-labeling of DNA strand breaks (TUNEL) for $60 \mathrm{~min}$ at $37^{\circ} \mathrm{C}$. Sections were then incubated with Converter-AP for $30 \mathrm{~min}$ at $37^{\circ} \mathrm{C}$ and alkaline phosphatase was visualized after 10-15 min of treatment with Fast Red TR/Naphthol AS-MX phosphate (Sigma), containing $1 \mathrm{mM}$ levamisole as an endogenous alkaline phosphatase inhibitor. Sections were counterstained with methyl green and mounted with Kaiser's glycerol jelly.

Computer-assisted image analysis. After immunohistochemical staining or TUNEL assay of sections from saline and drugtreated samples, images of fields were photographed with a Sony digital camera. Images from single sections were digitally recorded using a rectangular template and recordings were processed using Northern Eclipse image analysis software (50). Thresholds were set using green and red channels. The thresholds were determined, as described previously (50).

Statistical evaluation. Statistical analyses of tumor volume and weight data, from in vivo experiments, were conducted using data analysis tools in Microsoft Excel. To improve visual clarity, only the positive standard error value is shown for the tumor volume curves. Selected p-values for tumor weight comparisons are presented in the results section with complete statistical analyses available by request from the authors.

\section{Results}

Preliminary screening of GTI-2040 in combination with standard therapies in a murine xenograft model of tumor growth. Early screening studies with GTI-2040 were carried out on a broad range of cancers and a number of combinations with standard chemotherapeutic and immunotherapeutic drugs. For RCC, GTI-2040 was tested in combination with FU, vinblastine and IL-2 in murine xenograft tumor growth assays. All agents given as single therapies demonstrated a significant decrease in the growth of tumors compared to saline-treated controls and the combination with GTI-2040 resulted in improvement in the efficacy of test agents resulting in complete regression of tumors in all animals for all combinations (data not shown). The combination treatments were statistically significantly better than the respective single agent chemotherapy treatments and although combination treatment was reproducibly better than treatment with GTI2040 alone it was not statistically significant due in large part to effectiveness of the GTI-2040 treatment. GTI-2040 was administered at an optimal dose of $10 \mathrm{mg} / \mathrm{kg}$ every other day and this dose was previously shown to cause regression of 
A
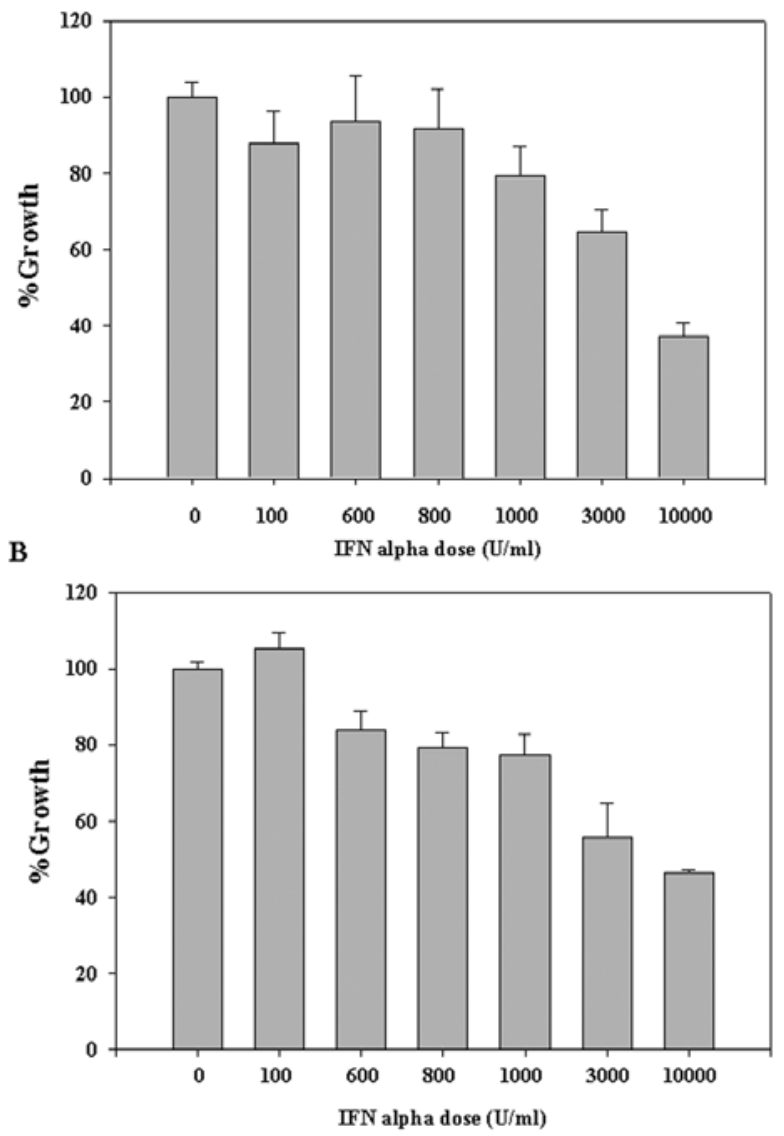

Figure 1. A498 (A) and Caki-1 (B) cells were treated with increasing concentrations of IFN $\alpha(0,100,600,800,1000,3000$ and $10000 \mathrm{U} / \mathrm{ml})$. After incubation for $96 \mathrm{~h}$, XTT reagent was added to each sample (prepared in triplicate) and absorbance at $490 \mathrm{nM}$ was determined for each

Caki-1 tumors in some but not all mice within a treatment group (47). In the GTI-2040 treatment groups 30-40\% of animals had complete regression of the implanted tumor, whereas in combination groups tumor regression exceeded $90 \%$ and in most cases reached $100 \%$. Although GTI-2040 cooperates with a number of standard chemotherapies there is little clinical evidence to support the use of chemotherapy for advanced RCC $(3,4,52)$. IL-2 is a standard option for treatment of advanced RCC, however clinical application is often limited by toxicity leading to only a small percentage of patients being eligible for treatment resulting in IFN $\alpha$ increasingly being used for treatment of $\operatorname{RCC}(7,8)$.

Caki-1 and A498 human renal carcinoma cell lines are sensitive to interferon a by in vitro proliferation assays. A number of studies have demonstrated that many RCC cell lines are sensitive to direct cytotoxic effects of IFN $\alpha$ including Caki-1 and A498 renal cell carcinoma cell lines (53-55). In order to confirm and compare the sensitivities of Caki-1 and A498 cell lines, in vitro cell proliferation assays were performed after treatment with IFN $\alpha$. The results shown in Fig. 1 demonstrate that both Caki and A498 cells are sensitive to IFN $\alpha$ and that the response appears to be dose-dependent at the higher doses of INF $\alpha(1000-10000 \mathrm{U} / \mathrm{ml})$. These data indicated that Caki-1 and A498 cell lines are relatively similar in their sensitivity to IFN $\alpha$ and would be amenable to assessment of combination effects with IFN $\alpha$ and GTI-2040 in vivo.

GTI-2040 cooperates with interferon $\alpha$ in two murine xenograft models of renal cell carcinoma tumor growth. Previously, GTI-2040 was shown to be a highly effective treatment in xenograft tumor growth models of human cancers, including two renal cell carcinoma models. At the highest concentration of GTI-2040 tested, $10 \mathrm{mg} / \mathrm{kg} / 48 \mathrm{~h}$, there was highly effective anti-tumor activity including complete regression in a significant number of test animals. In the current study, intermediate (or sub-optimal) doses of GTI-2040 and IFN $\alpha$ were tested in xenograft models of human renal tumor growth. Tumor growth was assessed by measurement of tumor volume over the course of the experiment as well as tumor weights taken at the experimental endpoint. Growth of Caki-1 and A498 human renal cell carcinoma cells implanted in SCID mice was effectively inhibited by both GTI-2040 and IFN $\alpha$ treatments (Figs. 2 and 3 , respectively). As shown, the response to these agents is dose-dependent with increasing dose resulting in improved anti-tumor activity. A number of dose combinations of these two agents resulted in anti-tumor activity that exceeded the effects of either agent alone. The following statistical analyses are for the tumor weights measured at the experimental endpoint shown in Figs. 2B and 3B. For Caki-1, the lowest doses of both agents had little effect on tumor growth either alone or in combination, but at the highest doses the single agents reduced tumor growth compared to saline although only GTI-2040 reached statistical significance $(\mathrm{p}=0.007)$. In addition, the combination treatment at the higher dose levels was significantly better than saline and GTI-2040 alone ( $\mathrm{p}=0.0003$ compared to saline, $\mathrm{p}=0.007$ compared to GTI2040). Although the high dose treatment was reproducibly superior to IFN $\alpha$ alone it did not meet significance ( $p=0.07)$, due in large part to the high variability in the IFN $\alpha$ high-dose mono-therapy. Although neither low dose GTI-2040 nor high dose IFN $\alpha$ demonstrated statistically significant anti-tumor efficacy alone $(\mathrm{p}=0.6$ and $\mathrm{p}=0.2$ compared to saline, respectively) the combination demonstrated better anti-tumor efficacy than either agent alone and reached significance compared to saline ( $\mathrm{p}=0.003$ compared to saline). Some regression was observed in the high-dose combination treatment group (5 of 10 animals).

A498 tumors were consistently more sensitive to treatment by all agents with the exception of the lowest dose of IFN $\alpha$ (Fig. 3A and B). For A498 tumors, there was significant antitumor activity of GTI-2040 and IFN $\alpha$ compared to saline at the higher dose levels $(\mathrm{p}=0.003, \mathrm{p}<0.001$, respectively). The combination with lower doses of IFN $\alpha$ and GTI-2040 did increase anti-tumor activity compared to the corresponding mono-therapies but it was not statistically significant compared to mono-therapy. Although neither agent as a mono-therapy at the lower doses demonstrated significant activity compared to saline, in combination significance was reached ( $\mathrm{p}=0.009$ compared to saline). There was significant improvement compared to mono-therapy at all higher dose IFN $\alpha$ combinations [ $\left(10^{5} \mathrm{IFN} \alpha+2.5 \mathrm{mg} / \mathrm{kg} / 48 \mathrm{~h}\right.$ GTI-2040: $\mathrm{p}=0.002$ compared to GTI-2040 alone, $\mathrm{p}<0.001$ compared 


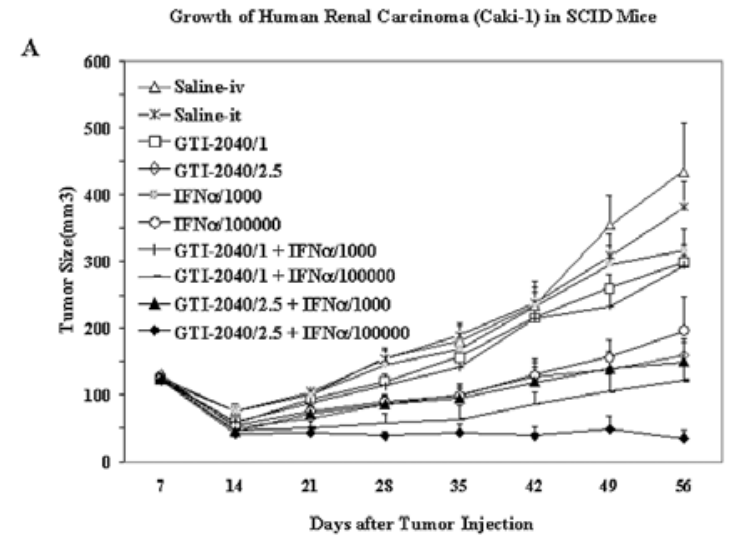

B

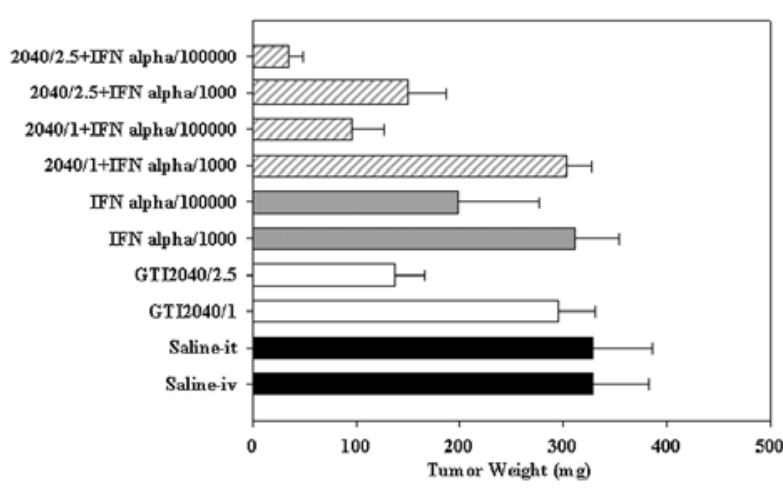

Figure 2. Caki-1 cells $\left(5 \times 10^{6}\right.$ cells in $\left.100 \mu 1 \mathrm{PBS}\right)$ were subcutaneously implanted into the right flank of SCID mice. After tumors reached an approximate volume of $100-150 \mathrm{~mm}^{3}, 7$ days after tumor cell injection, GTI-2040 and IFN $\alpha$, were administered. The control groups received saline administered as either intravenous or intratumoral injections. GTI-2040 was administered by bolus infusion into the tail vein every other day at either $1 \mathrm{mg} / \mathrm{kg}$ or $2.5 \mathrm{mg} / \mathrm{kg}$ doses in $0.1 \mathrm{ml}$ saline as indicated. IFN $\alpha$ was administered by intratumoral injections of either 1000 or 100000 units in $0.05 \mathrm{ml}$ saline as indicated. Treatment was for 5 days per week, for three weeks followed by a one week rest and then repeated 3 week treatment. Treatment was interrupted briefly to allow growth of tumors such that cooperative effects would be evaluable. The combination treatments are as indicated above for the single agent treatments. (A) Caliper measurements at 1 -week intervals were used to calculate tumor volumes. Each point represents mean tumor volume and standard error calculated from 10 animals per experiment. (B) Mice were sacrificed 57 days after tumor cell implantation and tumor weight for each was determined. Mean tumor weight and standard error (SE) are shown for each experimental group of 10 mice.

with IFN $\alpha$ alone $),\left(10^{5}\right.$ IFN $\alpha+1 \mathrm{mg} / \mathrm{kg} / 48 \mathrm{~h}$ GTI-2040: $\mathrm{p}=0.001$ compared to GTI-2040 alone, $\mathrm{p}=0.002$ compared with IFN $\alpha$ alone)]. Nine of 10 complete regressions were observed at the highest dose combination in A498 cells while neither single agent treatment resulted in tumor regressions. In addition, high-dose IFN $\alpha$ combined with the lower dose GTI-2040 also resulted in 5 of 10 regressions.

Although almost complete regression was achieved in A498 experiments shown in Fig. 3, this was not achieved for Caki-1 tumor growth (Fig. 2) at the initial concentrations tested. Shown in Fig. 4A are the results of Caki-1 experiments with an increased GTI-2040 dose in combination with the highest dose of IFN $\alpha$ used in Fig. 2. In this experiment, increasing the GTI-2040 dose to $5 \mathrm{mg} / \mathrm{kg} / 48 \mathrm{~h}$ resulted in complete regression of tumors in the combination group (10 of 10 animals) and statistically superior efficacy compared to
G GrowthofHuman Renal Carcinom (A998) in SCD Mice
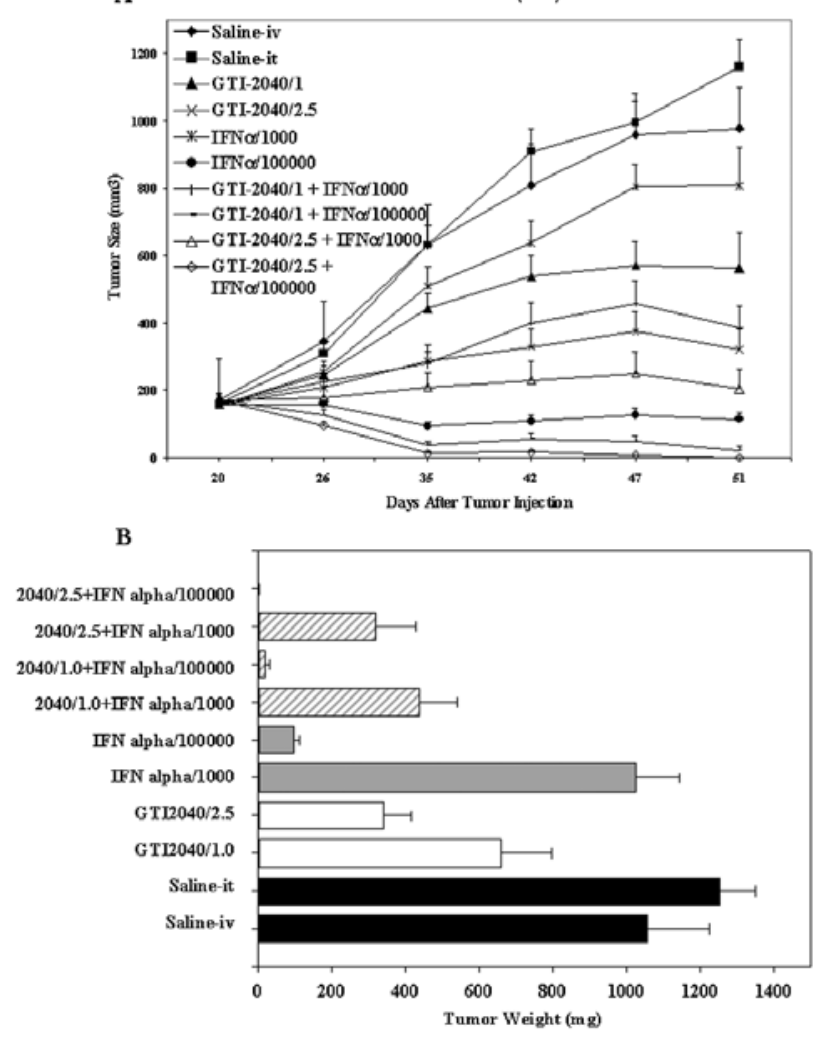

Figure 3. A498 cells (9x106 cells in $100 \mu 1$ PBS) were subcutaneously implanted into the right flank of SCID mice. After tumors reached an approximate volume of $100-150 \mathrm{~mm}^{3}, 20$ days after tumor cell injection, GTI-2040 and IFN $\alpha$, were administered. The control groups received saline administered as either intravenous or intratumoral injections. GTI-2040 was administered by bolus infusion into the tail vein every other day at either $1 \mathrm{mg} / \mathrm{kg}$ or $2.5 \mathrm{mg} / \mathrm{kg}$ doses in $0.1 \mathrm{ml}$ saline as indicated. IFN $\alpha$ was administered for 5 days per week by intratumoral injections of either 1000 or 100000 units in $0.05 \mathrm{ml}$ saline as indicated. IFN $\alpha$ treatment continued to experimental endpoint to assess maximal cooperative effect. The combination treatments are as indicated above for the single agent treatments. (A) Caliper measurements at the indicated intervals were used to calculate tumor volumes. Each point represents mean tumor volume calculated from 10 animals per experiment. (B) Mice were sacrificed 51 days after tumor cell implantation and tumor weight for each was determined. Mean tumor weight and standard error (SE) are shown for each experimental group of 10 mice.

both mono-therapies ( $\mathrm{p}=0.002$ compared to GTI-2040 alone and $\mathrm{p}=0.02$ compared to IFN $\alpha$ alone). GTI-2040 was effective at the higher dose and resulted in two of ten complete regressions. The results shown in Fig. 4B were from an experiment designed to better understand the intermediate effects of GTI-2040 and IFN $\alpha$ against A498 tumors. At an intermediate dose of IFN $\alpha$ the anti-tumor activity as a mono-therapy was approximately equivalent to GTI-2040 at $2.5 \mathrm{mg} / \mathrm{kg} / 48 \mathrm{~h}$. The combination of these doses clearly demonstrates the potential cooperative effects of this drug combination, as the combination was significantly better than either agent alone therapies ( $\mathrm{p}=0.001$ compared to GTI-2040 alone and $\mathrm{p}<0.001$ compared to IFN $\alpha$ alone).

Control oligonucleotide does not increase the anti-tumor activity of interferon $\alpha$. Phosphorothioated oligonucleotides (PS-ODNs) have been shown to have a number of non-specific effects related to protein binding and immunostimulatory 

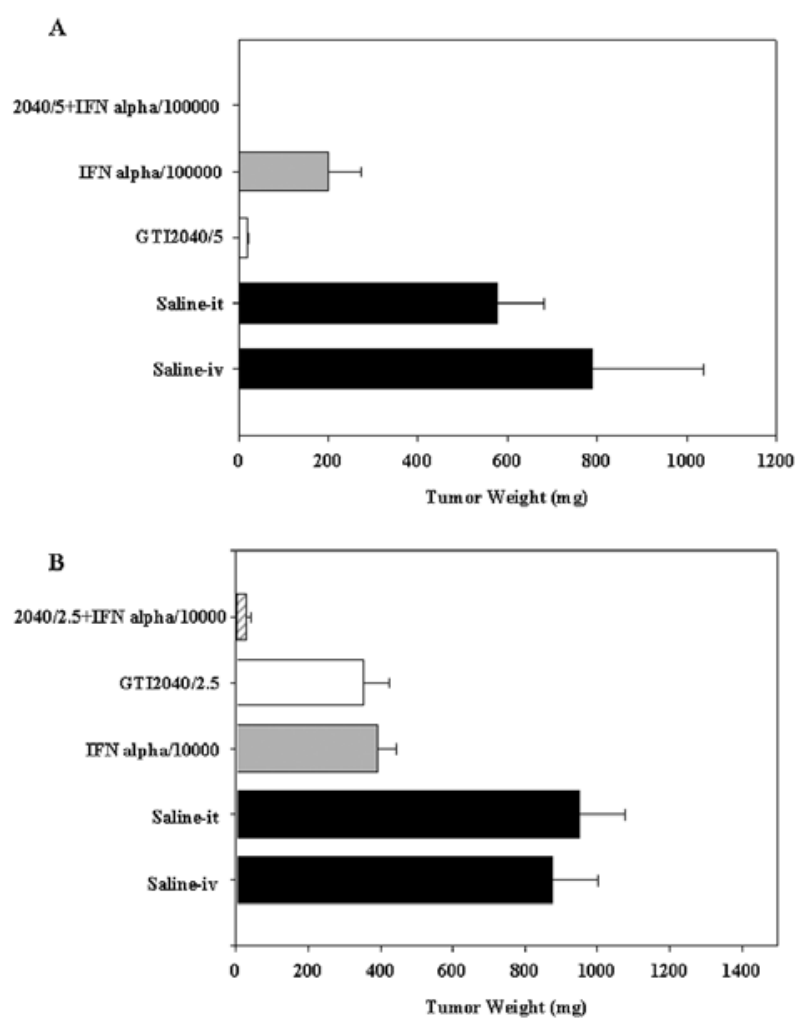

Figure 4. (A) Caki-1 cells ( $5 \times 10^{6}$ cells in $100 \mu 1$ PBS) were subcutaneously implanted into the right flank of SCID mice. After tumors reached an approximate volume of $100-150 \mathrm{~mm}^{3}, 7$ days after tumor cell injection, GTI-2040 and IFN $\alpha$, were administered. The control groups received saline administered as either intravenous or intratumoral injections. GTI-2040 was administered by bolus infusion into the tail vein every other day at $5 \mathrm{mg} / \mathrm{kg}$ doses in $0.1 \mathrm{ml}$ saline as indicated. IFN $\alpha$ was administered for 5 days per week by intratumoral injections of 100000 units in $0.05 \mathrm{ml}$ saline. IFN $\alpha$ treatment continued to experimental endpoint to assess maximal cooperative effect. The combination treatments are as indicated above for the single agent treatments. Mice were sacrificed 56 days after tumor cell implantation and tumor weight for each was determined. Mean tumor weight and standard error (SE) are shown for each experimental group of 10 mice. (B) A498 cells $\left(9 \times 10^{6}\right.$ cells in $100 \mu 1$ PBS) were subcutaneously implanted into the right flank of SCID mice. After tumors reached an approximate volume of $150 \mathrm{~mm}^{3}, 20$ days after tumor cell injection, GTI-2040 and IFN $\alpha$, were administered. The control groups received saline administered as either intravenous or intratumoral injections. GTI-2040 was administered by bolus infusion into the tail vein every other day at a $2.5 \mathrm{mg} / \mathrm{kg}$ dose in $0.1 \mathrm{ml}$ saline. IFN $\alpha$ was administered for 5 days per week by intratumoral injection of 10000 units in $0.05 \mathrm{ml}$ saline for two weeks. Treatment with IFN $\alpha$ stopped early to observe cooperativity at sub-optimal dosing. The combination treatments are as indicated above for the single agent treatments. Mice were sacrificed 45 days after tumor cell implantation and tumor weight for each was determined. Mean tumor weight and standard error (SE) are shown for each experimental group of 10 mice.

properties due to backbone and non-target sequences $(56,57)$. Published results from a previous study indicate that the antitumor effects of GTI-2040 are predominantly due to sequencespecific effects related to the down-regulation of the R2 target (47). In order to assess potential non-specific effects against renal xenograft models a control oligonucleotide containing the same base composition as GTI-2040 but with a scrambled sequence was assessed in the Caki-1 xenograft model. The results in Fig. 5 demonstrate that the control oligonucleotide, GTI-2040scr alone has no anti-tumor activity compared to saline $(\mathrm{p}=0.82)$ and does not improve the anti-tumor activity of IFN $\alpha$ ( $p=0.297$ compared to IFN $\alpha$ alone, $p=0.07$ compared

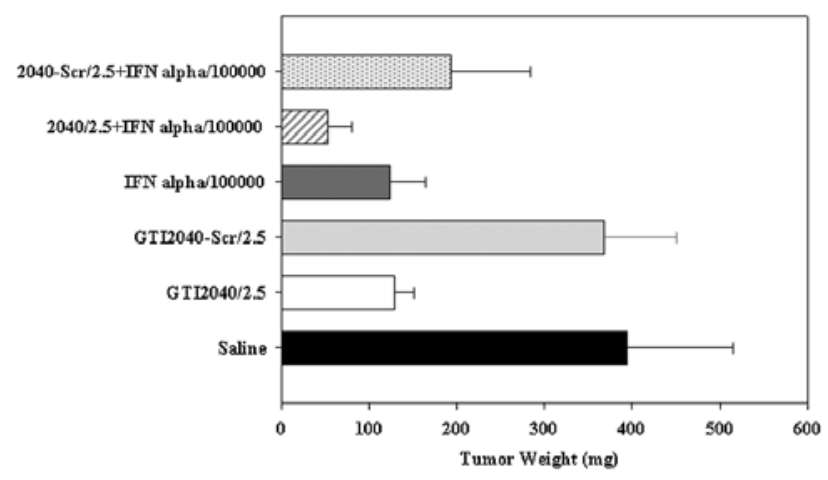

Figure 5. Caki-1 cells $\left(5 \times 10^{6}\right.$ cells in $100 \mu 1$ PBS $)$ were subcutaneously implanted into the right flank of SCID mice. After tumors reached an approximate volume of $100 \mathrm{~mm}^{3}, 7$ days after tumor cell injection, GTI-2040, GTI-2040Scr and/or IFN $\alpha$, were administered. The control groups received saline administered as either intravenous or intratumoral injections. GTI-2040 and GTI-2040Scr was administered by bolus infusion into the tail vein every other day at $2.5 \mathrm{mg} / \mathrm{kg}$ doses in $0.1 \mathrm{ml}$ saline. IFN $\alpha$ was administered for 5 days per week by intratumoral injections of 100000 units in $0.05 \mathrm{ml}$ saline. IFN $\alpha$ treatment continued to experimental endpoint to assess maximal cooperative effect. The combination treatments are as indicated above for the single agent treatments. Mice were sacrificed 56 days after tumor cell implantation and tumor weight for each was determined. Mean tumor weight and standard error (SE) are shown for each experimental group of 10 mice.

to GTI-2040scr alone). At the same ODN concentration GTI-2040 as a mono-therapy has anti-tumor activity ( $p=0.003$ ) and improves the effects of IFN $\alpha$ in combination ( $\mathrm{p}=0.005$ compared to IFN $\alpha \alpha$ alone). Interestingly IFN $\alpha$, at the same suboptimal concentration as Fig. 2 but given continuously without the one week break, demonstrated significant activity compared to saline reiterating the importance of both dose and schedule in designing future studies.

GTI-2040 treatment results in increased apoptosis and decreased proliferation of renal cell carcinoma cells xenografted into mice. The data presented above indicate that treatment with IFN $\alpha$ and GTI-2040 is highly effective as a combination therapy. Initial histochemical analyses were undertaken in order to understand the mechanism of anti-tumor activity and to assess whether the effects of GTI-2040 are due in part to immunostimulation, as has been observed with PS-ODNs containing a CpG motif in a specific hexameric context. Mice bearing Caki-1 tumor xenografts were treated as indicated in the figure legend to Fig. 6 and tumor samples were collected as described in the methods at the times indicated and analyzed for rate of proliferation and apoptosis. As shown in Fig. 6 and Table I, GTI-2040 as a monotherapy significantly decreases the rate of proliferation and increases the rate of apoptosis in tumor cells from xenograft samples $(\mathrm{p}<0.001$ for both analyses compared to saline). The intermediate sub-optimal dose of IFN $\alpha$ used in the study did not result in changes to either proliferation rate or apoptosis. The control ODN did not demonstrate any effects on proliferation or apoptosis when given as a single agent.

A combination of the GTI-2040 and IFN $\alpha$ resulted in an additional increase in apoptosis and a decrease in proliferative rate, suggesting that IFN $\alpha$ may have sensitized tumor cells to GTI-2040 [(Apoptosis: $\mathrm{p}<0.001$ compared to saline and IFN $\alpha$ alone and $\mathrm{p}=0.016$ compared to GTI-2040 alone) 
Table I. Rate of apoptosis and proliferation of renal cell carcinoma cells xenografted into mice.

\begin{tabular}{lrcrrrrr}
\hline Treatment & Saline (i.v.) & Saline (i.t.) & GTI-2040 & IFN $\alpha$ & GTI-2040scr & $\begin{array}{r}\text { GTI-2040scr } \\
+ \text { IFN } \alpha\end{array}$ & $\begin{array}{c}\text { GTI-2040 } \\
+ \text { IFN } \alpha\end{array}$ \\
\hline \% Apoptotic cells & $2.21 \pm 0.79$ & $2.23 \pm 0.8$ & $8.34 \pm 2.26$ & $2.25 \pm 0.92$ & $2.3 \pm 1.13$ & $2.83 \pm 1.21$ & $13.56 \pm 5.46$ \\
$\%$ BrdU-positive cells & $20.12 \pm 3.39$ & $19.70 \pm 2.56$ & $12.11 \pm 2.19$ & $20.16 \pm 2.51$ & $20.87 \pm 2.21$ & $13.95 \pm 3.85$ & $7.25 \pm 1.99$ \\
\hline
\end{tabular}

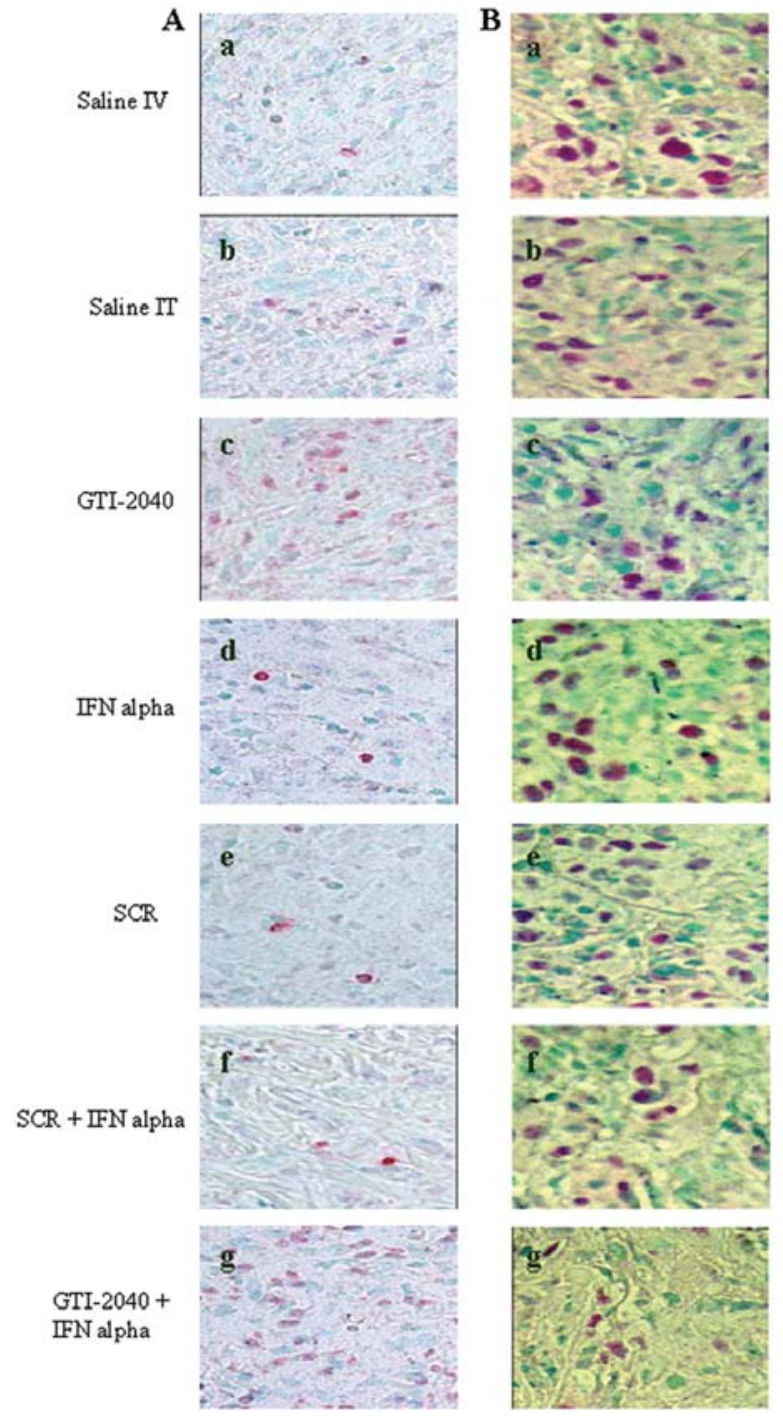

Figure 6. Caki-1 cells $\left(5 \times 10^{6}\right.$ cells in $100 \mu 1$ PBS $)$ were subcutaneously implanted into the right flank of SCID mice. After tumors reached an approximate volume of $100 \mathrm{~mm}^{3}, 7$ days after tumor cell injection, GTI-2040 and/or IFN $\alpha$, were administered. The control groups received saline administered as either intravenous or intratumoral injections. GTI-2040 was administered by bolus infusion into the tail vein every other day at a $5 \mathrm{mg} / \mathrm{kg}$ dose in $0.1 \mathrm{ml}$ saline. IFN $\alpha$ was administered for 5 days per week by intratumoral injections of 100000 units in $0.05 \mathrm{ml}$ saline. The combination treatments are as indicated above for the single agent treatments. Treatment was suspended after an initial two week treatment period and then resumed after a two week rest to ensure tumor sample availability at the experimental endpoint. Mice were sacrificed 45 days after tumor cell implantation and tumor samples were prepared for BrdU analysis and immunohistochemistry. (A) Tumor samples prepared and analyzed for rate of apoptosis as described in the Methods. Treatments were as follows: a, Saline (i.v.); b, Saline (i.t.); c, GTI-2040; d, IFN $\alpha$; e, GTI-2040Scr; f, GTI-2040Scr + IFN $\alpha$; g, GTI-2040 + IFN $\alpha$. (B) Tumor samples prepared and analyzed for rate of proliferation as described in the Methods. Treatments were as follows: a, Saline (i.v.); b, Saline (i.t.); c, GTI-2040; d, IFN $\alpha$; e, GTI-2040Scr; f, GTI-2040Scr + IFN $\alpha$; g, GTI-2040 + IFN $\alpha$.
(Proliferation: $\mathrm{p}<0.0001$ for all comparisons)]. This would be consistent with a number of studies that demonstrate that IFN $\alpha$ induces and/or sensitizes cells to apoptosis $(58,59)$. The control scrambled oligonucleotide had no effect on either apoptosis or proliferation as a single agent but did result in a combined effect with IFN $\alpha$ when measuring proliferation ( $p=0.001$ compared to both saline and IFN $\alpha$ alone). This is likely due to the known non-specific effects of PS-ODNs as there was not a concomitant increase in apoptosis. Although the combined effect was significantly better than the monotherapy groups it was not as good at decreasing proliferation as the combination with GTI-2040 ( $p<0.001$ comparing the two combinations).

Although the SCID mice used in this study are deficient in $\mathrm{T}$ and $\mathrm{B}$ cell function tumor samples were assessed for macrophage and NK cell infiltration into tumors upon treatment with either agent and in combination. There were no observable NK or macrophage infiltrates in the tumor samples analyzed suggesting that the mechanism of action observed is not the result of immune stimulation (not shown). Tumor infiltrates have been demonstrated in GTI-2040-treated CD-1 mice (intact $B$ cell function) suggesting that although immune stimulation can be a contributing factor in GTI-2040 antitumor activity it is not involved in the effects seen in SCID mice used for these studies (data not shown). These results are consistent with previous studies that demonstrated efficacy in SCID beige mice lacking NK cell function in addition to $\mathrm{B}$ and $\mathrm{T}$ cell deficiency (47).

\section{Discussion}

A number of in vitro and in vivo studies have demonstrated a role for RNR in progression and metastasis of cancer (introduction above). Previous studies demonstrated that inhibiting expression of the R2 subunit with GTI-2040 results in significant anti-tumor activity measured in human xenograft models in mice (47). Renal cell carcinoma tumors implanted into mice demonstrated high sensitivity to GTI-2040 treatment resulting in regression of some tumors at high doses of GTI-2040 (47). The results presented here provide compelling pre-clinical evidence in support of combination therapy for renal cell carcinoma using GTI-2040. Although GTI-2040 treatment enhanced the anti-tumor efficacy of every drug tested, the most notable results from a clinical perspective were with IFN $\alpha$ and IL-2, both immunological agents that have become standard therapies for advanced RCC $(5,6)$. Furthermore, the cooperative effects were sequence-specific since treatment with scrambled ODN did not result in enhanced action of interferon $\alpha$. The broad-spectrum cooperative activity of GTI-2040 may be a reflection of RNR inhibition leading to a decrease in the rate of proliferation and increased apoptosis 
observed in the histochemistry and immunohistochemistry analyses. The observed effect of the scrambled oligonucleotide on proliferation in combination with IFN $\alpha$ but not on apoptosis or as a single agent is likely the result of previously described non-specific effects of PS-ODN. Control PS-ODNs can exhibit minor biological activity in vitro and in vivo, but usually at lower efficacy than observed for the specific PS-ODN [examples (60-62)]. This is usually related to immunostimulatory or serum protein binding properties of the PS-ODNs $(56,57)$.

For a number of reasons including; consistent response rate, tolerability and ease of administration, IFN $\alpha$ has become the preferred agent for comparison in phase III trials and for evaluation of emerging drug candidates either as single agents or in combination with standard therapies $(7,8)$. As a result, the current study focused on IFN $\alpha$ as a comparator drug and as the combination treatment for GTI-2040. IFN $\alpha$ has been extensively studied in pre-clinical models of cancer progression. IFN $\alpha$ is a biological agent that has diverse functions and may act as an anti-tumor agent via multiple direct and indirect pathways including immune stimulation of acquired and innate immune effector cells, direct antiproliferative effects on tumor cells, induction of apoptosis and attenuation of tumor angiogenesis $(58,59,63-67)$. The detailed molecular mechanism by which the combination of GTI-2040 and IFN $\alpha$ induces apoptosis and decreases proliferation remains to be elucidated and may involve multiple and/or overlapping pathways. Given what is known about R2 and its role in tumorigenesis there exist a number of pathways by which these agents cooperate. Future studies will attempt to elucidate the molecular components of the combined effect of IFN $\alpha$ and GTI-2040 with an emphasis on whether the cooperative effects observed are the result of over-lapping or non-overlapping molecular pathways. Of particular interest and warranting further analysis is that an intermediate suboptimal dose of IFN $\alpha$ did not increase apoptosis or decrease proliferation in RCC tumor cells on its own, but the combination with GTI-2040 resulted in sensitization of RCC tumor cells to both increased apoptosis and decreased proliferation. In this study, IFN $\alpha$ demonstrated dose-dependent inhibition of proliferation in vitro and so further studies are necessary to determine whether higher doses of IFN $\alpha$ affect proliferation and apoptosis in vivo. IFNa is known to induce and or sensitize cells to apoptosis via a number of pathways including JAK/STAT and PI3 signaling and involving activation of multiple caspases and proapoptotic molecules (58). In addition, recent studies on RCC and hepatocellular carcinoma cells with IFN $\alpha$ in combination with 5-FU implicate p53 in the synergy demonstrated by these compounds $(68,69)$. Both of the RCC cell lines studied here are wild-type for p53 (ATCC) and so additional studies with p53 mutant cell lines or by expression of a dominant-negative p53 in Caki-1 and A498 cells may be useful in determining whether p53 is involved in the synergy observed with GTI-2040. Separate from its role as a subunit of RNR R2 has been implicated in tumor progression via interaction with a number of oncogenes including synergism with the Ras pathway $(31,32)$. Given that the EGF/Ras/Raf-1/Erk survival pathway has been shown to counteract the effects of IFN $\alpha$, there is a compelling possibility that inhibition of R2 may augment
IFN $\alpha$-mediated anti-tumor effects via down-regulation of the Ras/Raf 1 signalling pathway $(58,70-72)$.

Although IFN $\alpha$ was administered intratumorally in the current study, additional studies will address the potential for alternative modes of administration. Preliminary studies suggest that systemic administration of IFN $\alpha$ (subcutaneous on opposite flank) in the mouse model of human RCC may be effective in decreasing tumor growth both as a single agent and in combination with GTI-2040. Further studies are required to establish optimal dosing and to compare the therapeutic benefit to intratumoral administration.

Immunotherapy has been standard treatment for RCC for many years but more recently there is a growing list of mechanism-based drugs that have promising therapeutic activity based on an increased understanding of how RCC develops and progresses. Given the current paradigm in cancer management it is expected that these new drugs will be used in combination with each other and/or with immunotherapy. As research expands the knowledge of how RCC differs between patients the hope is that the combination therapy will be tailored to individual or sub-groups of patients that stand to benefit most from each therapy or combination. Given that GTI-2040 has the potential to cooperate with both chemotherapy and immunotherapy it may also prove useful with newer treatments. For this reason the GTI-2040 research program will be expanded to include these emerging therapies.

GTI-2040 has been evaluated in a number of clinical trials and has demonstrated reasonable tolerability both as a single agent and in combination with chemotherapeutic agents $(48,73)$. Although limited to early stages of clinical investigation there is accumulating evidence that GTI-2040 specifically down-regulates the R2 target and may have therapeutic activity (74). The data presented here and preliminary animal studies on a number of indications and combinations (our unpublished observations) support the future use of GTI-2040 in combination with standard drugs in clinical studies.

\section{Acknowledgements}

We are grateful to Robert Peralta for technical assistance.

\section{References}

1. American Cancer Society. Facts and Figures, 2008.

2. Motzer RJ, Mazumdar M, Bacik J, Berg W, Amsterdam A and Ferrara J: Survival and prognostic stratification of 670 patients with advanced renal cell carcinoma. J Clin Oncol 17: 2530-2540, 1999.

3. Hartmann JT and Bokemeyer C: Chemotherapy for renal cell carcinoma. Anticancer Res 19: 1541-1553, 1999.

4. Yagoda A, Petrylak D and Thompson S: Cytotoxic chemotherapy for advanced renal cell carcinoma. Urol Clin North Am 20: 303-321, 1993.

5. Bleumer I, Oosterwijk E, De Mulder P and Mulders PF: Immunotherapy for renal cell carcinoma. Eur Urol 44: 65-75, 2003.

6. Nathan PD and Eisen TG: The biological treatment of renal-cell carcinoma and melanoma. Lancet Oncol 3: 89-96, 2003.

7. Motzer RJ, Bacik J, Murphy BA, Russo P and Mazumdar M: Interferon-alfa as a comparative treatment for clinical trials of new therapies against advanced renal cell carcinoma. J Clin Oncol 20: 289-296, 2002.

8. Mickisch GH: Rational selection of a control arm for randomized trials in metastatic renal cell carcinoma. Eur Urol 43: 670-679, 2003. 
9. Brugarolas J: Renal-cell carcinoma - molecular pathways and therapies. N Engl J Med 356: 185-187, 2007.

10. Mellado B and Gascon P: Molecular biology of renal cell carcinoma. Clin Transl Oncol 8: 706-710, 2006.

11. Motzer RJ and Bukowski RM: Targeted therapy for metastatic renal cell carcinoma. J Clin Oncol 24: 5601-5608, 2006.

12. Escudier B: Advanced renal cell carcinoma: current and emerging management strategies. Drugs 67: 1257-1264, 2007.

13. Longo R, D'Andrea MR, Sarmiento R, Salerno F and Gasparini G: Integrated therapy of kidney cancer. Ann Oncol 18 Suppl 6: vi141-148, 2007

14. Cory JG: Purine and pyrimidine nucleotide metabolism. In: Biochemistry with Clinical Correlations. Devlin T (ed). 4th edition. Wiley-Liss, New York, pp489-523, 1997.

15. Jordan A and Reichard P: Ribonucleotide reductases. Annu Rev Biochem 67: 71-98, 1998.

16. Thelander M, Graslund A and Thelander L: Subunit M2 of mammalian ribonucleotide reductase. Characterization of a homogeneous protein isolated from M2-overproducing mouse cells. J Biol Chem 260: 2737-2741, 1985.

17. Larsson A and Sjoberg BM: Identification of the stable free radical tyrosine residue in ribonucleotide reductase. EMBO J 5: 2037-2040, 1986.

18. von Dobeln U and Reichard P: Binding of substrates to Escherichia coli ribonucleotide reductase. J Biol Chem 251 : 3616-3622, 1976.

19. Engstrom Y, Eriksson S, Jildevik I, Skog S, Thelander L and Tribukait B: Cell cycle-dependent expression of mammalian ribonucleotide reductase. Differential regulation of the two subunits. J Biol Chem 260: 9114-9116, 1985.

20. Eriksson S, Gudas LJ, Clift SM, Caras IW, Ullman B and Martin DW Jr: Evidence for genetically independent allosteric regulatory domains of the protein M1 subunit of mouse ribonucleotide reductase. J Biol Chem 256: 10193-10197, 1981.

21. Mann GJ, Musgrove EA, Fox RM and Thelander L: Ribonucleotide reductase M1 subunit in cellular proliferation, quiescence, and differentiation. Cancer Res 48: 5151-5156, 1988

22. Thelander L and Reichard P: Reduction of ribonucleotides. Annu Rev Biochem 48: 133-158, 1979.

23. Eriksson S, Graslund A, Skog S, Thelander L and Tribukait B: Cell cycle-dependent regulation of mammalian ribonucleotide reductase. The $\mathrm{S}$ phase-correlated increase in subunit M2 is regulated by de novo protein synthesis. J Biol Chem 259 11695-11700, 1984

24. Eriksson S and Martin DW Jr: Ribonucleotide reductase in cultured mouselymphoma cells. Cell cycle-dependent variation in the activity of subunit protein M2. J Biol Chem 256: 9436-9440, 1981

25. Nakano K, Balint $E$, Ashcroft $M$ and Vousden $K H_{\text {: }} A$ ribonucleotide reductase gene is a transcriptional target of p53 and p73. Oncogene 19: 4283-4289, 2000.

26. Tanaka H, Arakawa H, Yamaguchi $\mathrm{T}$, et al: A ribonucleotide reductase gene involved in a p53-dependent cell-cycle checkpoint for DNA damage. Nature 404: 42-49, 2000

27. Yamaguchi T, Matsuda K, Sagiya Y, Iwadate M, Fujino MA, Nakamura Y and Arakawa H: p53R2-dependent pathway for DNA synthesis in a p53-regulated cell cycle checkpoint. Cancer Res 61: 8256-8262, 2001 .

28. Guittet O, Hakansson P, Voevodskaya N, et al: Mammalian p53R2 protein forms an active ribonucleotide reductase in vitro with the R1 Protein, which is expressed both in resting cells in response to DNA damage and in proliferating cells. J Biol Chem 276: 40647-40651, 2001.

29. Elford HL, Freese M, Passamani E and Morris HP: Ribonucleotide reductase and cell proliferation. I. Variations of ribonucleotide reductase activity with tumor growth rate in a series of rat hepatomas. J Biol Chem 245: 5228-5233, 1970.

30. Weber G: Biochemical strategy of cancer cells and the design of chemotherapy: G. H. A. Clowes Memorial Lecture. Cancer Res 43: 3466-3492, 1983.

31. Fan H, Villegas C, Huang A and Wright JA: The mammalian ribonucleotide reductase $\mathrm{R} 2$ component cooperates with a variety of oncogenes in mechanisms of cellular transformation. Cancer Res 58: 1650-1653, 1998.

32. Fan H, Villegas C and Wright JA: Ribonucleotide reductase R2 component is a novel malignancy determinant that cooperates with activated oncogenes to determine transformation and malignant potential. Proc Natl Acad Sci USA 93: 14036-14040, 1996.
33. Huang A, Fan H, Taylor WR and Wright JA: Ribonucleotide reductase $\mathrm{R} 2$ gene expression and changes in drug sensitivity and genome stability. Cancer Res 57: 4876-4881, 1997.

34. Kuo ML, Hwang HS, Sosnay PR, Kunugi KA and Kinsella TJ: Overexpression of the R2 subunit of ribonucleotide reductase in human nasopharyngeal cancer cells reduces radiosensitivity. Cancer J 9: 277-285, 2003.

35. Zhou BS, Hsu NY, Pan BC, Doroshow JH and Yen Y: Overexpression of ribonucleotide reductase in transfected human KB cells increases their resistance to hydroxyurea: M2 but not M1 is sufficient to increase resistance to hydroxyurea in transfected cells. Cancer Res 55: 1328-1333, 1995.

36. Zhou BS, Tsai P, Ker R, et al: Overexpression of transfected human ribonucleotide reductase M2 subunit in human cancer cells enhances their invasive potential. Clin Exp Metastasis 16: 43-49, 1998.

37. Goan YG, Zhou B, Hu E, Mi S and Yen Y: Overexpression of ribonucleotide reductase as a mechanism of resistance to 2,2defluorodeoxycyctidine in the human KB cancer cell line. Cancer Res 59: 4204-4207, 1999.

38. Chen S, Zhou B, He F and Yen Y: Inhibition of human cancer cell growth by inducible expression of human ribonucleotide reductase antisense cDNA. Antisense Nucleic Acid Drug Dev 10: 111-116, 2000

39. Shao J, Zhou B, Chu B and Yen Y: Ribonucleotide reductase inhibitors and future drug design. Curr Cancer Drug Targets 6: 409-431, 2006.

40. Barker CA, Burgan WE, Carter DJ, et al: In vitro and in vivo radiosensitization induced by the ribonucleotide reductase inhibitor Triapine (3-aminopyridine-2-carboxaldehydethiosemicarbazone). Clin Cancer Res 12: 2912-2918, 2006.

41. Novotny L, Rauko P, Liska J, Elford HL and Szekeres T: Potentiation of the activity of cisplatin and cyclophosphamide by trimidox, a novel ribonucleotide reductase inhibitor, in leukemia-bearing mice. Cancer Lett 233: 178-184, 2006.

42. Sigmond J, Kamphuis JA, Laan AC, Hoebe EK, Bergman AM and Peters GJ: The synergistic interaction of gemcitabine and cytosine arabinoside with the ribonucleotide reductase inhibitor triapine is schedule dependent. Biochem Pharmacol 73: 1548-1557, 2007.

43. Yee KW, Cortes J, Ferrajoli A, et al: Triapine and cytarabine is an active combination in patients with acute leukemia or myelodysplastic syndrome. Leuk Res 30: 813-822, 2006.

44. Duxbury MS, Ito H, Benoit E, Zinner MJ, Ashley SW and Whang EE: Retrovirally mediated RNA interference targeting the M2 subunit of ribonucleotide reductase: A novel therapeutic strategy in pancreatic cancer. Surgery 136: 261-269, 2004.

45. Duxbury MS, Ito H, Zinner MJ, Ashley SW and Whang EE: RNA interference targeting the M2 subunit of ribonucleotide reductase enhances pancreatic adenocarcinoma chemosensitivity to gemcitabine. Oncogene 23: 1539-1548, 2004.

46. Crooke ST: Progress in antisense technology. Annu Rev Med 55: 61-95, 2004.

47. Lee Y, Vassilakos A, Feng N, et al: GTI-2040, an antisense agent targeting the small subunit component (R2) of human ribonucleotide reductase, shows potent antitumor activity against a variety of tumors. Cancer Res 63: 2802-2811, 2003

48. Desai AA, Schilsky RL, Young A, et al: A phase I study of antisense oligonucleotide GTI-2040 given by continuous intravenous infusion in patients with advanced solid tumors. Ann Oncol 16: 958-965, 2005.

49. Pagliaro LC, Perez CA, Tu SM and Daliani DD: Phase II study of capecitabine single-agent therapy in patients with metastatic renal cell carcinoma. Urol Oncol 24: 487-491, 2006.

50. Miao D, Bai X, Panda D, McKee M, Karaplis A and Goltzman D: Osteomalacia in hyp mice is associated with abnormal phex expression and with altered bone matrix protein expression and deposition. Endocrinology 142: 926-939, 2001

51. Miao D, He B, Karaplis AC and Goltzman D: Parathyroid hormone is essential for normal fetal bone formation. J Clin Invest 109: 1173-1182, 2002.

52. Fossa SD, Martinelli G, Otto U, et al: Recombinant interferon alfa-2a with or without vinblastine in metastatic renal cell carcinoma: results of a European multi-center phase III study. Ann Oncol 3: 301-305, 1992.

53. Eisenkraft BL, Nanus DM, Albino AP and Pfeffer LM: alphaInterferon down-regulates epidermal growth factor receptors on renal carcinoma cells: relation to cellular responsiveness to the antiproliferative action of alpha-interferon. Cancer Res 51: $5881-5887,1991$ 
54. Nanus DM, Pfeffer LM, Bander NH, Bahri S and Albino AP: Antiproliferative and antitumor effects of alpha-interferon in renal cell carcinomas: correlation with the expression of a kidney-associated differentiation glycoprotein. Cancer Res 50: 4190-4194, 1990.

55. Rohde D, Hayn HK, Blatter J and Jakse G: The efficacy of 2',2'difluorodeoxycytidine (gemcitabine) combined with interferon in human renal cell carcinoma cell lines. Int J Oncol 12: 1361-1366, 1998

56. Krieg AM: Immune effects and mechanisms of action of $\mathrm{CpG}$ motifs. Vaccine 19: 618-622, 2001.

57. Monteith DK, Henry SP, Howard RB, Flournoy S, Levin AA, Bennett CF and Crooke ST: Immune stimulation-a class effect of phosphorothioate oligodeoxynucleotides in rodents. Anticancer Drug Design 12: 421-432, 1997.

58. Caraglia M, Marra M, Pelaia G, Maselli R, Caputi M, Marsico SA and Abbruzzese A: Alpha-interferon and its effects on signal transduction pathways. J Cell Physiol 202: 323-335, 2005 .

59. Sangfelt and Strander H: Apoptosis and cell growth inhibition as antitumor effector functions of interferons. Med Oncol 18: 3-14, 2001.

60. Uchida T, Gao JP, Wang C, et al: Antitumor effect of bcl-2 antisense phosphorothioate oligodeoxynucleotides on human renal-cell carcinoma cells in vitro and in mice. Mol Urol 5: 71-78, 2001.

61. Yamanaka K, Rocchi P, Miyake H, Fazli L, Vessella B, Zangemeister-Wittke $U$ and Gleave ME: A novel antisense oligonucleotide inhibiting several antiapoptotic Bcl-2 family members induces apoptosis and enhances chemosensitivity in androgen-independent human prostate cancer PC3 cells. Mol Cancer Ther 4: 1689-1698, 2005.

62. Yip KW, Mocanu JD, Au PY, et al: Combination bcl-2 antisense and radiation therapy for nasopharyngeal cancer. Clin Cancer Res 11: 8131-8144, 2005.

63. Dinney CP, Bielenberg DR, Perrotte P, Reich R, Eve BY, Bucana $C D$ and Fidler IJ: Inhibition of basic fibroblast growth factor expression, angiogenesis, and growth of human bladder carcinoma in mice by systemic interferon-alpha administration. Cancer Res 58: 808-814, 1998.
64. Ozawa S, Shinohara H, Kanayama HO, et al: Suppression of angiogenesis and therapy of human colon cancer liver metastasis by systemic administration of interferon-alpha. Neoplasia 3: 154-164, 2001.

65. Pfeffer LM, Dinarello CA, Herberman RB, et al: Biological properties of recombinant alpha-interferons: 40th anniversary of the discovery of interferons. Cancer Res 58: 2489-2499, 1998.

66. von Marschall Z, Scholz A, Cramer T, et al: Effects of interferon alpha on vascular endothelial growth factor gene transcription and tumor angiogenesis. J Natl Cancer Inst 95: 437-448, 2003.

67. Xu D, Erickson S, Szeps M, et al: Interferon alpha down-regulates telomerase reverse transcriptase and telomerase activity in human malignant and nonmalignant hematopoietic cells. Blood 96: 4313-4318, 2000.

68. Fujioka N, Ariyasu T, Arai N, et al: Role of p53 in the inhibitory effects of interferon-alpha subtypes on proliferation of hepatocellular carcinoma cells. Biomed Res 27: 219-226, 2006.

69. Shang D, Ito N, Watanabe J, Awakura Y, Nishiyama H, Kamoto T and Ogawa O: Synergy of Interferon-alpha and 5-Fluorouracil in Human Renal Cell Carcinoma Requires p53 Activity. Eur Urol 22: 22, 2007.

70. Bruzzese F, Di Gennaro E, Avallone A, et al: Synergistic antitumor activity of epidermal growth factor receptor tyrosine kinase inhibitor gefitinib and IFN-alpha in head and neck cancer cells in vitro and in vivo. Clin Cancer Res 12: 617-625, 2006.

71. Caraglia M, Abbruzzese A, Leardi A, et al: Interferon-alpha induces apoptosis in human KB cells through a stress-dependent mitogen activated protein kinase pathway that is antagonized by epidermal growth factor. Cell Death Differ 6: 773-780, 1999.

72. Caraglia M, Tagliaferri $\mathrm{P}$, Marra M, et al: EGF activates an inducible survival response via the RAS-> Erk-1/2 pathway to counteract interferon-alpha-mediated apoptosis in epidermoid cancer cells. Cell Death Differ 10: 218-229, 2003.

73. Stadler WM, Desai AA, Quinn DI, et al: A Phase I/II study of GTI-2040 and capecitabine in patients with renal cell carcinoma. Cancer Chemother Pharmacol 61: 689-694, 2007.

74. Klisovic RB, Blum W, Wei W, et al: Phase I study of GTI2040 , an antisense to ribonucleotide reductase, in combination with high-dose cytarabine in patients with acute myeloid leukemia. Clin Cancer Res 14: 3889-3895, 2008. 